\title{
Dependence of the micro-arcsecond metrology (MAM) testbed performance prediction on white light algorithm approach
}

TsePyng Janice Shen, Joseph Catanzarite, Mark H. Milman, Gregory W. Neat

TsePyng Janice Shen, Joseph Catanzarite, Mark H. Milman, Gregory W. Neat, "Dependence of the micro-arcsecond metrology (MAM) testbed performance prediction on white light algorithm approach," Proc. SPIE 4852, Interferometry in Space, (26 February 2003); doi: 10.1117/12.460921

SPIE Event: Astronomical Telescopes and Instrumentation, 2002, Waikoloa, Hawai'i, United States 


\title{
The dependence of the Micro-Arcsecond Metrology (MAM) Testbed performance prediction on white light algorithm approach
}

\author{
TsaePyng Janice Shen ${ }^{\mathrm{a}^{*}}$, Joe Catanzarite ${ }^{\mathrm{b}}$, Mark Milman $^{\mathrm{a}}$, Greg Neat ${ }^{\mathrm{a}}$ \\ a Jet Propulsion Laboratory, California Institute of Technology \\ ${ }^{b}$ Interferometry Science Center, California Institute of Technology
}

\begin{abstract}
MAM (Micro-Arcsecond Metrology Testbed), is a dedicated systems-level testbed that combines the major SIM (Space Interferometry Mission) subsystems including laser metrology, pointing, and pathlength control. The testbed is configured as a modified Michelson interferometer for the purpose of studying the white-light (science or guide stars) fringe measurement processes.
\end{abstract}

This paper will compare the performance of various algorithms using the MAM data, and will aid in our recommendation of how the SIM flight system should process the science and guide interferometer data.

The experiment measures optical pathlengths via the white-light phase and infrared (IR) laser metrology. The variance of phase differences between white light and metrology is directly related to our accuracy in relative optical pathlength measurements.

The full aperture He-Ne laser light ${ }^{1,2}$ served as a proxy to measure the optical path reference for white light in the system prior to the installation of SAVV (Sub-Aperture Vertex-to-Vertex ${ }^{3}$ ) 1.3 um laser metrology. In this simplified configuration, analysis of the white-light detected on the CCD and the He-Ne light detected on the photodiode was providing us with the phase differences between the two.

In general, MAM data are subject to random noise, e.g. shot noise and vibration, and systematic noise, e.g. drifts and cyclic errors, from instruments and detectors. Our noise floor in phase (or pathlength) difference measurements is determined by analysis of both the full aperture He-Ne laser light and white light fringe detected on the $\mathrm{CCD}^{4}$. Several fringe estimation algorithms have been developed and tested with the MAM data. Preprocessing procedures for fringe data have also been developed in conjunction with estimation algorithms to further enhance the fidelity of data ${ }^{1,2}$. The performance improvements are quantified with respect to a baseline algorithm, which is the least-squares fitting solution ${ }^{5}$. The adopted performance metrics are long-time modified Allan variance ${ }^{6}$ and short-time chop variance. The algorithms recommended to SIM are based on the improvements and sensitivity studies from MAM data analysis.

Keywords: white light algorithms, Space Interferometry Mission, Micro-Arcsecond Metrology testbed, microarcsecond astrometry, picometer

\section{Introduction}

MAM-1 testbed overview and its objectives were covered by the paper in this conference by Brad Hines ${ }^{3}$. The current objective of MAM is to provide a systems level demonstration of the $3 \mu$ as SIM narrow angle requirement. MAM experiments measure the optical pathlengths via the white-light phase and IR laser metrology. Phase delay differences between white light and metrology provide a measure of the optical path difference (OPD); performance metrics are based on modified Allan variance ${ }^{6}$ and chop variance ${ }^{7}$ of the OPD.

The MAM testbed is designed to validate that when all the picometer components, which include IR metrology, Test-Article (TA) and Inverse Interferometer Pseudo-Star (IIPS) optics, are put into the system

\footnotetext{
*tjs@huey.jpl.nasa.gov
} 
(i.e. MAM-1 testbed), the system performance still meets our picometer requirements. Fig.1 depicts the current configuration of the MAM testbed. Both HeNe and white light sources are available from IIPS. The MAM metric is the error between the IR metrology (SAVV) and the star light sensor (CCD), measuring identical optical paths. The phase delay of HeNe with respect to white light provides the measurement of our noise floor ${ }^{4}$.

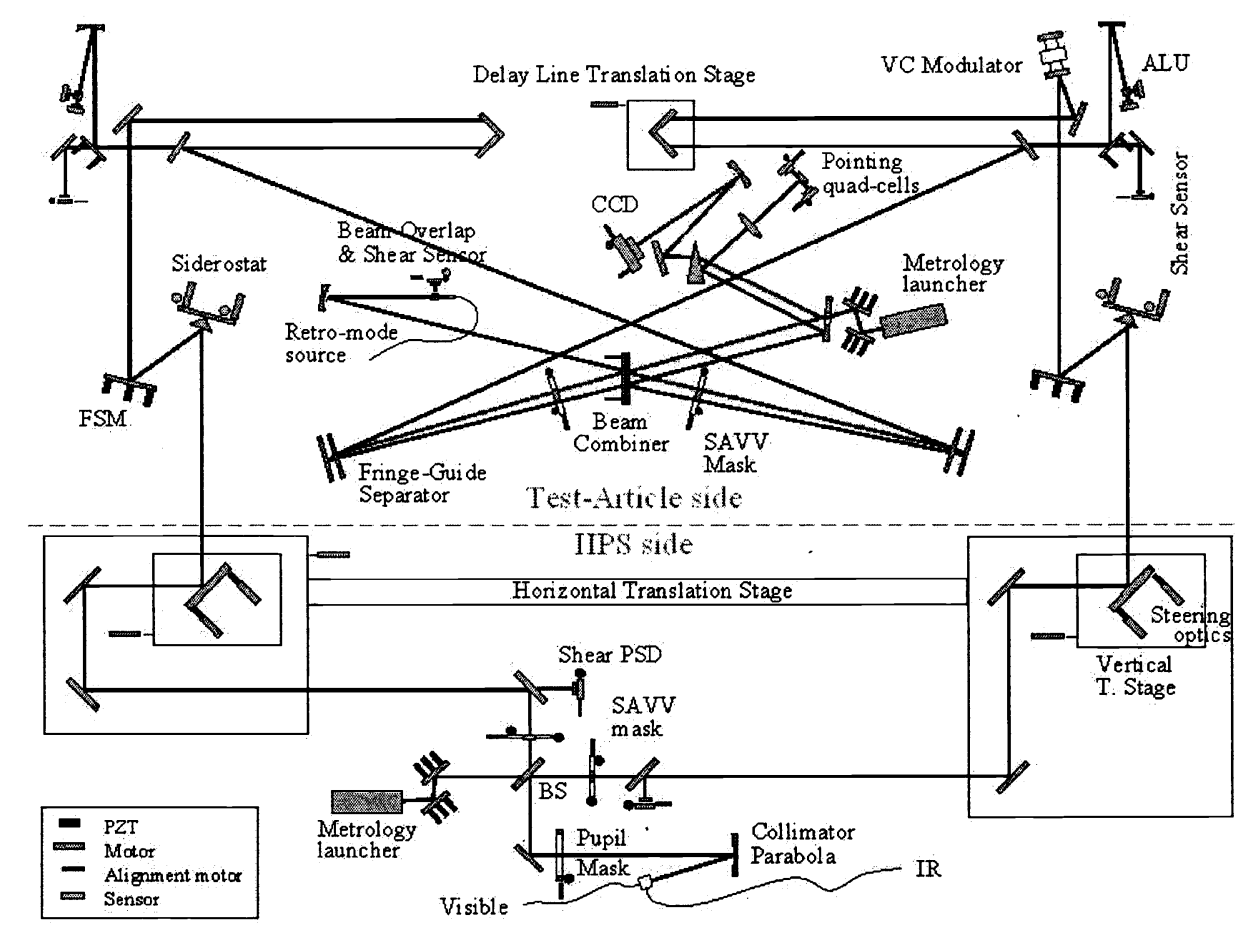

Fig. 1 MAM-1 current configuration.

\section{Fringe Model}

For the MAM testbed, white light fringes are detected by modulating the optical path via a voice coil in a triangle wave pattern. MAM has the ability to measure the positions of the modulation waveform at picometer accuracy by internal SAVV metrology at $1 \mathrm{KHz}$ rate. The recorded fringe intensity data at each triangle waveform is determined by our CCD camera recording speed, which is 500 frames/sec for our current configuration. For a $25 \mathrm{~Hz}$ triangle waveform, we record $20 \mathrm{CCD}$ frames (or time bins) during one triangle waveform. We average 2 samples of metrology data to match our camera data. The dither positions are the corresponding averaged positions of SAVV at each CCD frame.

$\mathrm{HeNe}$ and white light (which serves as proxy for the star-light) are spectrally dispersed linearly in wave number by the dispersion prism ("spectrometer") onto the 40x40 CCD camera. A single recorded frame from a $40 \times 3$ sub-region of the $40 \times 40$ camera is shown in Fig. 2. White light is dispersed nearly uniformly between 16th $(710 \mathrm{~nm})$ through 40 th $(950 \mathrm{~nm})$ pixels and $\mathrm{HeNe}$ is centered at left on 4th pixel. Dispersed white light on CCD has peak intensity around $30^{\text {th }}$ pixel, which reflects the intensity peak of our white light source. 


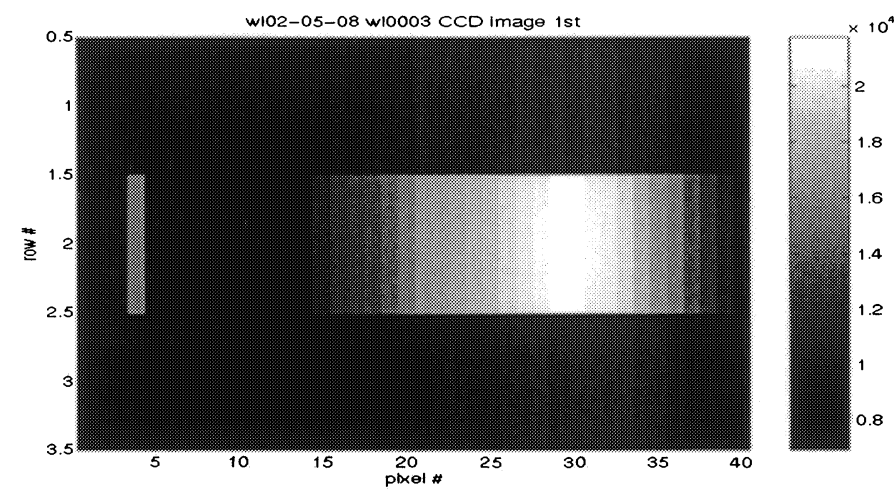

Fig.2. Dispersed fringe image on the CCD camera showing white light between $16^{\text {th }}(710 \mathrm{~nm})$ through $40^{\text {th }}$ $(950 \mathrm{~nm})$ pixels and $\mathrm{HeNe}$ at left centered on $4^{\text {th }}$ pixel.

For each recorded CCD frame, we co-add the recorded intensity of three rows at each pixel for analysis. Each white light pixel covers between $7.5 \mathrm{~nm}$ and $11 \mathrm{~nm}$ of spectral width and hence can be fit for quasimonochromatic fringes (see eq.(1)). A new fringe-phase measurement is produced every $40 \mathrm{~ms}$ for the voice-coil modulator scanning at $25 \mathrm{~Hz}$. A typical HeNe fringe from the CCD and its corresponding dither positions from SAVV are shown in Fig. 3. The vibrations and drifts of TA/IIPS, detector noise as well as SAVV noise all will appear in our recorded fringes and hence limit our accuracy in phase delay (or OPD) estimate.

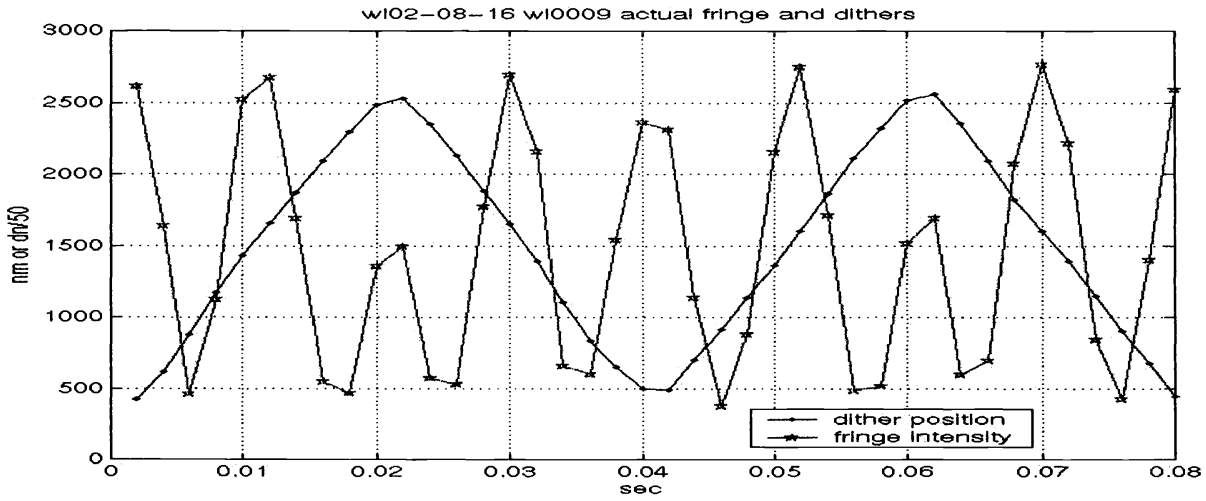

Fig. 3 Actual fringe and dither positions observed in MAM testbed.

Other realizations of fringe plots are shown in Fig.4. For HeNe fringe (left picture), we had further summed between pixels 2 to 7 in addition to coadding 3 rows on the recorded CCD frame. Each column in Fig. 4 covers $40 \mathrm{msec}$ time span and has 10 dithers for up stroke and 10 dithers for down stroke. 70,000 strokes are plotted along the horizontal axis, showing good stability over one hour. For the white light fringes (right picture), we displayed only one $40 \mathrm{msec}$ time stroke, as a function of the spectral channel along the horizontal axis. Nearly no tilt of the white light fringe across spectral pixels indicates that we are at nearly equal path between two interferometer arms during this run. 

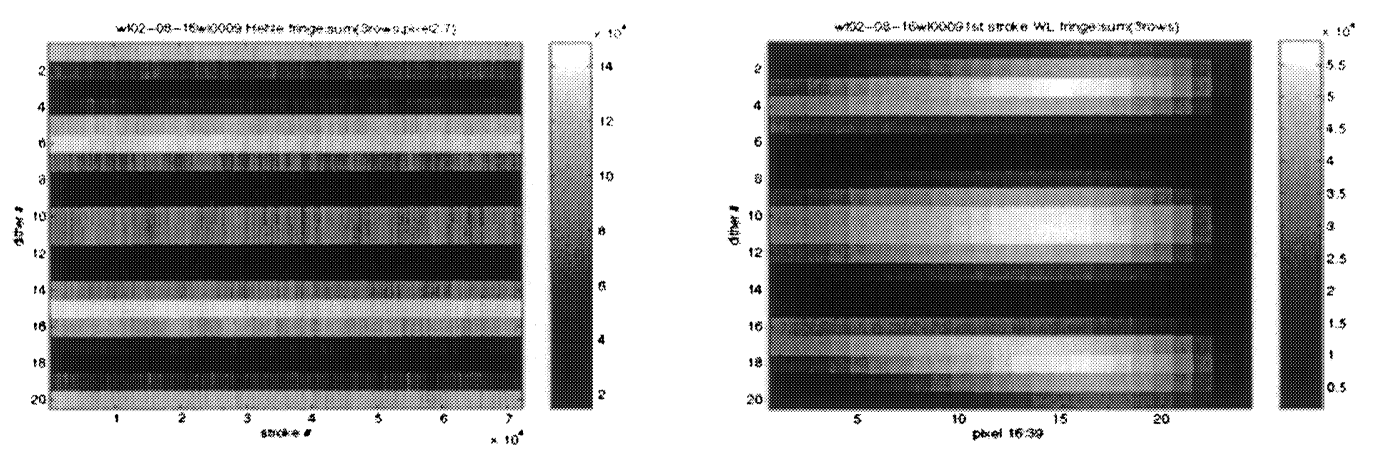

Fig. 4. HeNe (left) and white light (right) fringes from MAM testbed

The interference intensity model we used in our algorithms at a given stroke (or scan) is given by

$$
I_{i j l}^{d a t a}=I_{j l}\left\{1+V_{j l} \cos \left(k_{j l} x_{i j}+C_{j l}\right)\right\}
$$

where $l$ is the index for spectral pixels, $j$ is the index for strokes (or scans), $i$ is the index for dither positions within the stroke. For white light, $l=16, \ldots 39$, which is the spectral pixel index on CCD. $\boldsymbol{I}_{j l}, \boldsymbol{V}_{j l}, \boldsymbol{k}_{j l}$ and $\boldsymbol{C}_{j l}$ are fitted intensity, visibility, wave number and unknown phase (or delays*wavenumber), respectively, and all assumed to be constant over a given stroke, i.e. over all dither positions $\boldsymbol{x}_{i j}$ at given $j, l$.

Eq. (1) does not include detector noise, power fluctuation or alignment instability within a given stroke. Parameters in Eq. (1) can be categorized into system parameters and calibration parameters. These parameters are subject to errors due to light source fluctuations, thermal/tip-tilt/shear drift, vibration, cyclic error, or alignments. The error sources are dominant at different time scales and may be identified by our diagnostic metric. System parameters are intensity, visibility and unknown phase and will be solved by different algorithms. Calibration parameters are pre-calculated white light wave number at each spectral pixel, and measured dither positions.

\section{Diagnostics/performance metrics}

Allan variance ${ }^{6}\left(\sigma_{\mathrm{y}}{ }^{2}\right)$ is also known as two-sample variance. It is defined as $1 / 2$ of the time mean squares of the differences between successive readings of the data integrated over the integrating period $\tau$ and given by

$$
\sigma_{\mathrm{y}}^{2}(\tau)=1 / 2\left(<\left(\Delta \mathrm{y}_{\mathrm{i}}\right)^{2}>\right),
$$

where $\Delta y_{i}=\left\{y_{i+1}-y_{i}\right\}$; averaged samples $y_{i}$ are taken with no dead-time between them. Fig. 5 illustrates how the signal is spliced into integration intervals. The $y_{i}$ is the average of the data $y$ within the ith period $\tau$. In Eq, (2), "1/2" makes Allan variance equal to classical variance if the data are taken from white noise. $\sigma_{y}$ is the square root of Allan variance and is called Allan deviation. Allan variance has been widely used as a diagnostic tool in frequency standards ${ }^{6}$. Our data y are time series of delay phase (or optical pathlength) differences between white light and metrology. 


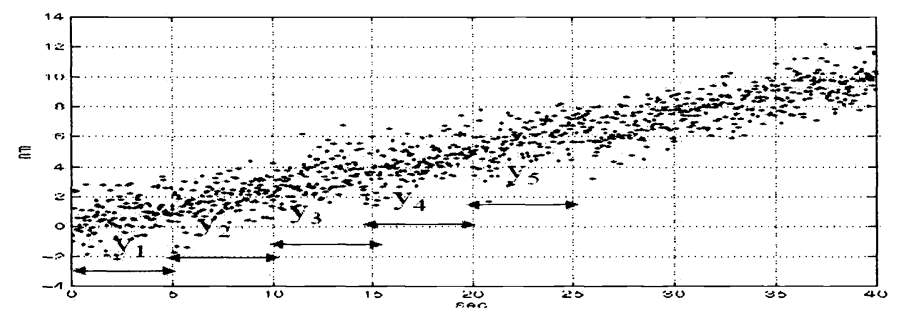

Fig. 5 Sample averages used in Allan variance or deviation.

For the MAM data analysis, we have adopted a modified Allan deviation ${ }^{6}$ and chop deviation ${ }^{7}$ for our diagnostics/performance metrics, based on the observation scenario for SIM chopping back and forward between reference and target stars ${ }^{7}$. The observation scenario representing a single SIM narrow angle observation involves observing a target star (one suspected to have orbiting planets) for 300 seconds, slewing to one or more reference stars, and observing them for 300 seconds total. The desired quantity is the difference in delays between the target star and the reference star(s). In the MAM testbed, we measure all the delays with respect to internal SAVV metrology.

The observation errors are subject to random and systematic (cyclic or drift) noise. Averaging reduces the random errors. It does not matter whether the integration time is contiguous or broken up into discrete time periods. Cyclic errors can be reduced by cyclic averaging or steady pathlength control. Chopping enables the reduction of drift errors. This approach assumes each field point sees the same time dependent error. The drift in the reference star measurement on either side (in time) of a target star measurement is then used to estimate the drift in the target star. This estimate is then removed from the target star value. The chop time is defined by the time constants of the drifts. The chop time should be long enough to minimize random errors and short compared to drift time constant in order to remove drift contributions effectively. The number of allowed chops is determined by the chosen chop time and allowable $300 \mathrm{sec}$ total integration time.

The modified Allan deviation, also called RT deviation, is defined as the time standard deviation of differences between successive readings of the $R$ (reference) and $T$ (target) data integrated over the integrating period $\tau$ and is given by

$$
\sigma_{\mathrm{yRT}}(\tau)=\operatorname{std}\left(\Delta \mathrm{y}_{\mathrm{i}}\right)
$$

where $\Delta y_{i}=\left\{y_{i+1}-y_{i}\right\}$ with $y_{i}=R$ (or $T$ ) and $y_{i+1}=T$ (or R), respectively. Samples of $R$ and $T$ are, respectively, the averaged delays of reference star and target star over the integration period and taken with no dead time between them in RT deviation. We have eliminated the factor of " $1 / 2$ " used in Eq.(3). RT deviation will be our diagnostic tool in MAM and SIM for field independent errors. An example of RT deviation is given in Fig. 6 . We indeed observed the long time stability improvements when we turn on the star trackers in addition to pathlength control to stabilize pointing. The drift time constant is on the order of few hundred sec. The minimal of RT deviation is reached around $30 \mathrm{sec}$. Note that the last data point is always close zero by definition (see Eq.(3)). 


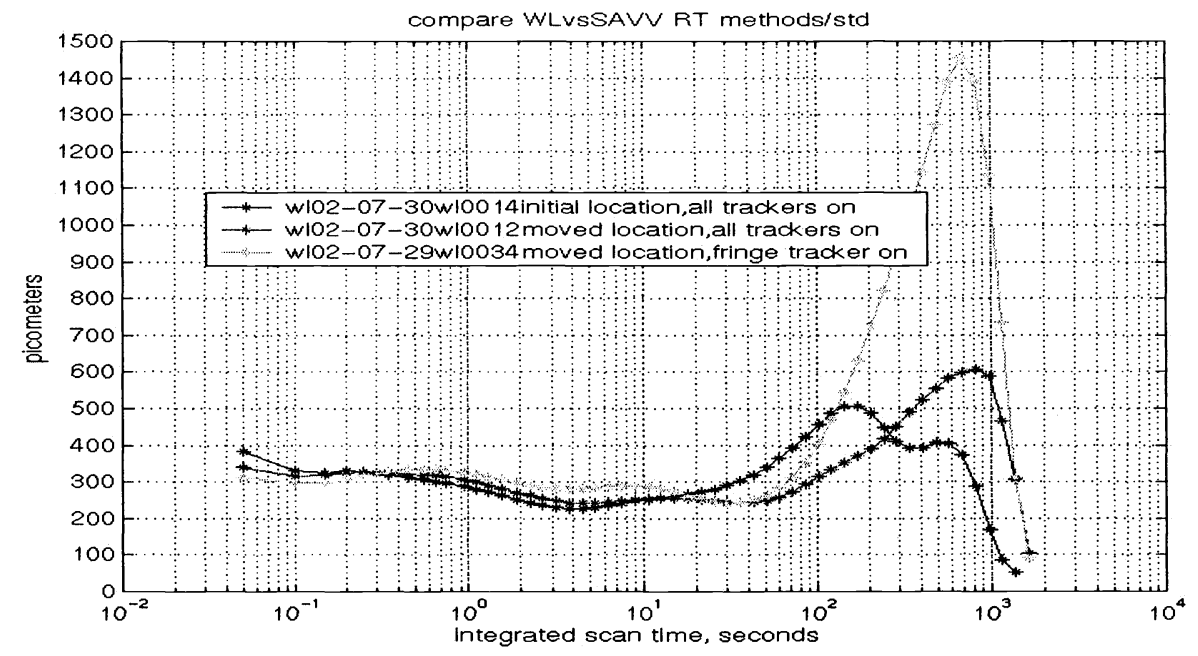

Fig. 6 Example of modified Allan deviation from MAM testbed

Chop deviation (also known as RT chop deviation) is defined as the time standard deviation of the differences between successive $\mathrm{R}$ (reference) and $\mathrm{T}$ (target) readings of the delays integrated over the period $\tau$ with dead time (or slew time) $t$ and is given by

$$
\sigma_{\text {ychop }}(\tau)=\operatorname{std}\left(\Delta y_{\text {ichop }}\right),
$$

where $y_{\text {ichop }}=R_{i}-T$ is the $1^{\text {st }}$ difference between the averaged delays of target and reference stars during integration period.

Fig. 7 depicts the sample averages used in our chopping analysis. Chopping makes SIM immune to many "drift" type error sources. Chop deviation vs. number of chops at a chosen integration time is a predictor of performance, not a diagnostic tool for identifying error sources. In our chopping analysis of quasi-static tests, we adopted an integration time of $\tau=30 \mathrm{sec}$ on target and reference, and a dead "slew" time of $t=15$ sec between target and reference measurements to be consistent with SIM requirements. We have 10 chops with $30 \mathrm{sec}$ integration time within $300 \mathrm{sec}$ observation time.

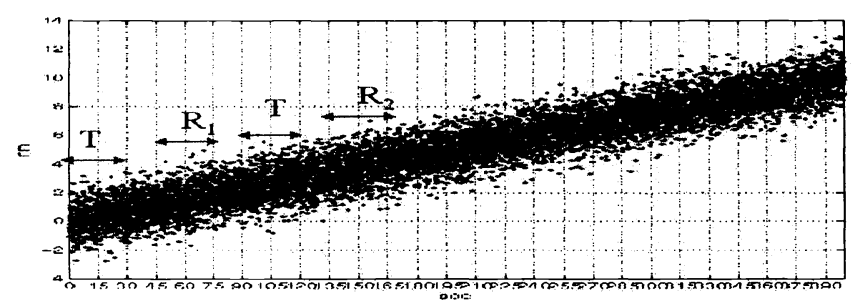

Fig. 7 Sample averages used in chop variance or deviation 


\section{Algorithms}

Phase delay estimation algorithms tested at current MAM testbed are least squares algorithm 5 , "optimal" spectrum algorithm ${ }^{8}$ and "optimal" CCD algorithm". The least squares algorithm (also called the pseudoinverse algorithm) is our baseline algorithm. "Optimal" spectrum algorithm (or $\mathrm{H}$ inverse algorithm) provides phase delays that are less sensitive to wave number errors." The phase delays solved by "optimal" $\mathrm{CCD}$ algorithm are less sensitive to shot and read noise on CCD at low light levels.

The white light phase delay is calculated from the mean of equal weighted phase delays at each spectral pixel and is equal to $\left\langle\mathrm{C}_{j l} * \lambda_{l} / 2 \pi\right\rangle$ where $\lambda_{l}$ are calibrated wavelength at $l$ 'th pixel. Measurement accuracy is determined by the accuracy of our OPD, which is the difference between $\left\langle\mathrm{C}_{j l}{ }^{*} \lambda_{1} / 2 \pi\right\rangle$ and phase measured by SAVV at each stroke. We obtain the white light delay estimate at each stroke using the phase delay rather than group delay, since the available spectral bandwidth of our CCD is narrow in our current MAM setup. The ratio of standard deviation between the group delay estimate and phase delay estimate is proportional to the ratio $\left\langle 2 \pi / \lambda_{l}>/ \Delta\left(2 \pi / \lambda_{l}\right)\right.$, viz. mean wave number $\left\langle 2 \pi / \lambda_{l}>\right.$ over usable bandwidth $\Delta\left(2 \pi / \lambda_{l}\right)$, of white light recorded on the $\operatorname{CCD}^{1}$. The advantage of phase delay estimates over group delay estimates is the improvement in SNR of pathlength measurement accuracy (or precision) without increased sensitivity to errors in the calibrated wave number.

\section{Results}

A comparison between least squares algorithm and "optimal" spectrum algorithm is shown in Fig. 7. Note the robustness of OPD estimations to wavelength errors provided by the $\mathrm{H}$ inverse algorithm. However, since our wavelength calibrations are good to a few $\mathrm{nm}$, the baseline algorithm provides adequate phase delay accuracy.
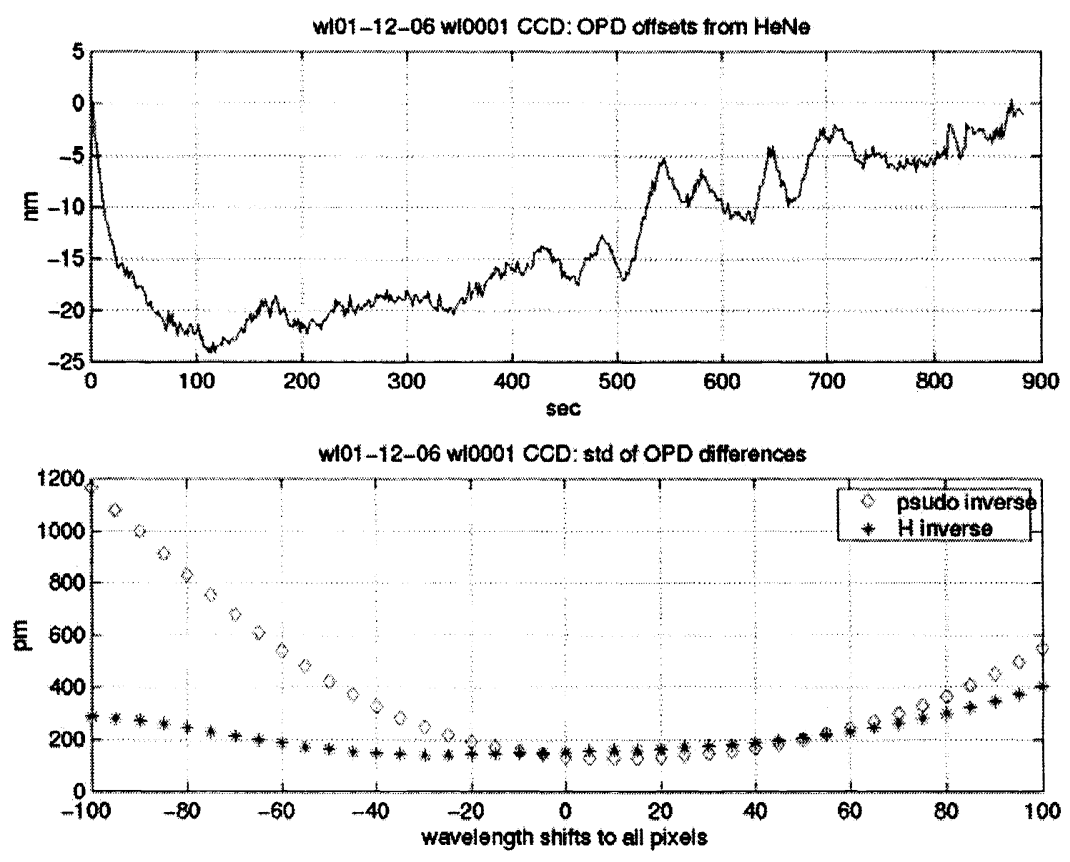

Fig 7. Optimal spectrum algorithm result

In the following figures, we present system level quasi-static (or field independent) tests performed on July 23,2002 . This was a one-hour long test with voice coil running a $20-\mathrm{Hz}$ triangle modulation waveform in 
open loop. We also positioned a pupil mask to minimize the photo-decenter between SAVV and star light ${ }^{8}$. The recorded $\mathrm{HeNe}$ and white light fringes are shown in Fig. 8. We can easily see that there is a total optical path change at the beginning of this run from HeNe fringes (left picture). We are not at equal path between two arms during this run, since the white light fringes (right picture) did tilt across spectral pixels
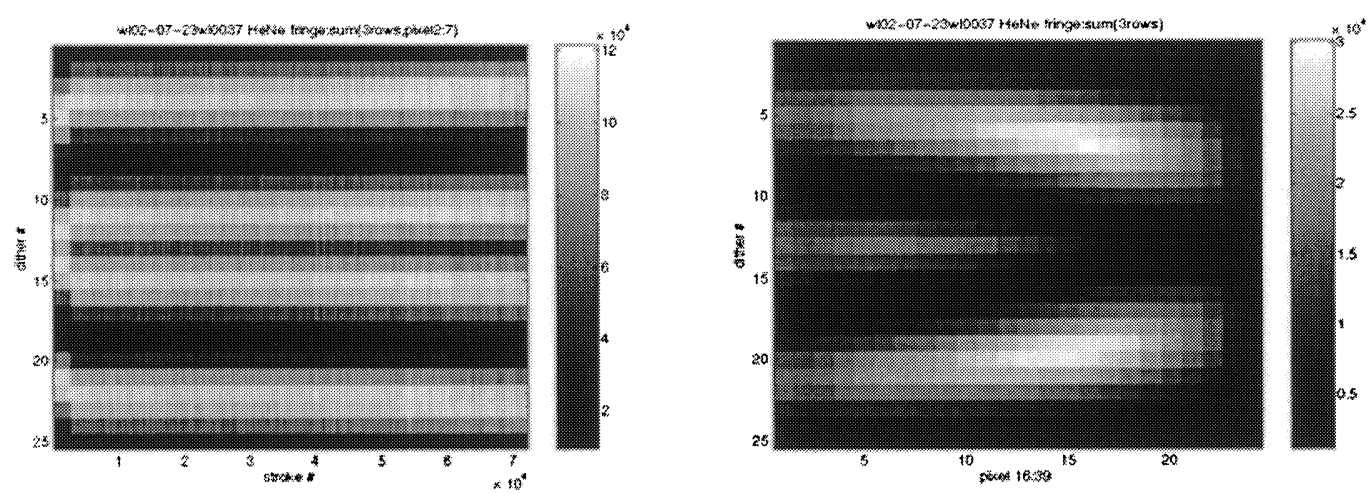

Fig. $8 \mathrm{HeNe}$ fringes and white light fringes recorded on July 23,2002 .

The OPD differences between HeNe, white light and SAVV are shown in Fig. 9. In the left figure, one can see that OPD differences between $\mathrm{HeNe}$ and SAVV (or HeNe vs SAVV) indeed possess good rejection ratio of 200 , viz. reducing from $\sim 80 \mathrm{~nm}$ total optical path caused by mechanical jump at the beginning of this one hour data run down to less than a nanometer. In the right figure, one can notice all three sensors (SAVV, $\mathrm{HeNe}$ and white light) agree relatively to each other at the nanometer level.
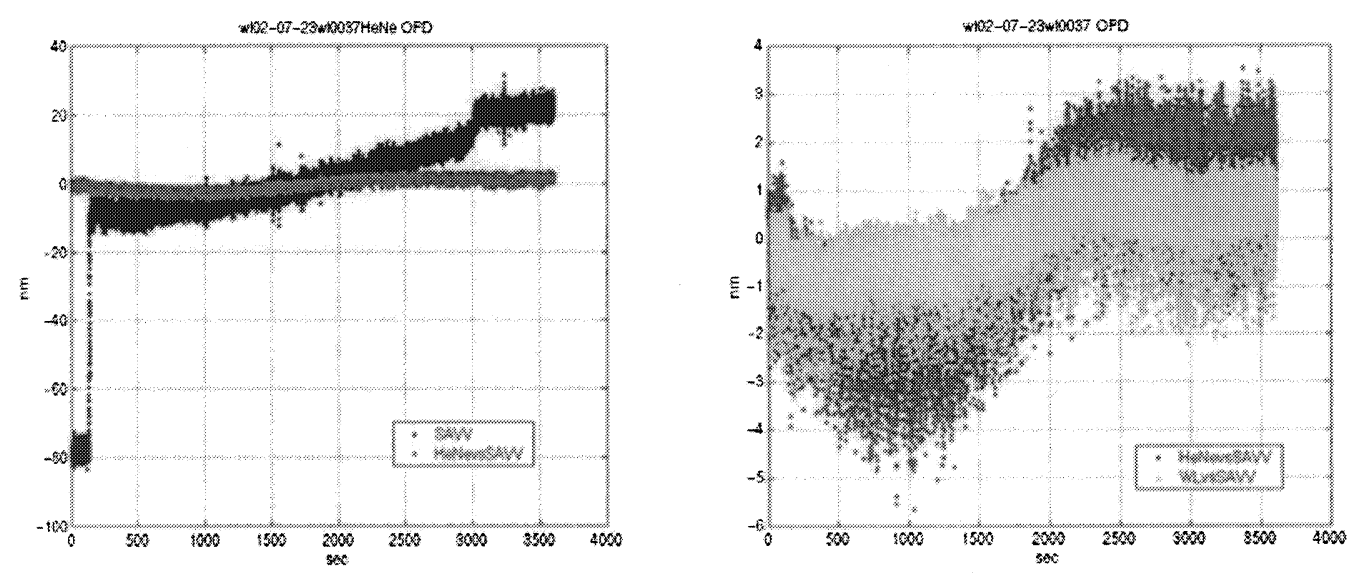

Fig. 9. OPD of SAVV (left), OPD differences between HeNe and SAVV (left/right), and OPD differences between white light and SAVV (right) for fringes shown in Fig. 8.

Metrics for the July 23 data are shown in Fig.10. At short integration time, random noise dominates. For long integration time, system drifts become important. In left figure, we explored the white light phase delay's behavior at different light levels. For white light fringes, the total light levels are directly proportional to the number of pixels used in analysis. From the Allan deviation plot on the left, we see that using one pixel rather than 24 pixels only affects the phase delays for integration times below a second or so, where the CCD noise dominates. As integration time increases beyond a second, the modified Allan deviation keeps decreasing till $\sim 50$ second. Beyond 50 second, the Allan deviation comes back up, since the 
system drifts come in at this time scale. As integration time approaches half hour, we have very few samples available for Eq.(3) and hence the values are artificially low near half hour integration time. Comparing different light levels on white light phase delays for the photon level in MAM, only short time differs and similar performance is achieved at $30 \mathrm{sec}$ integration. Hence the performance for chop deviation (right figure) of $30 \mathrm{sec}$ integration time is insensitive to light level at our current MAM setup.
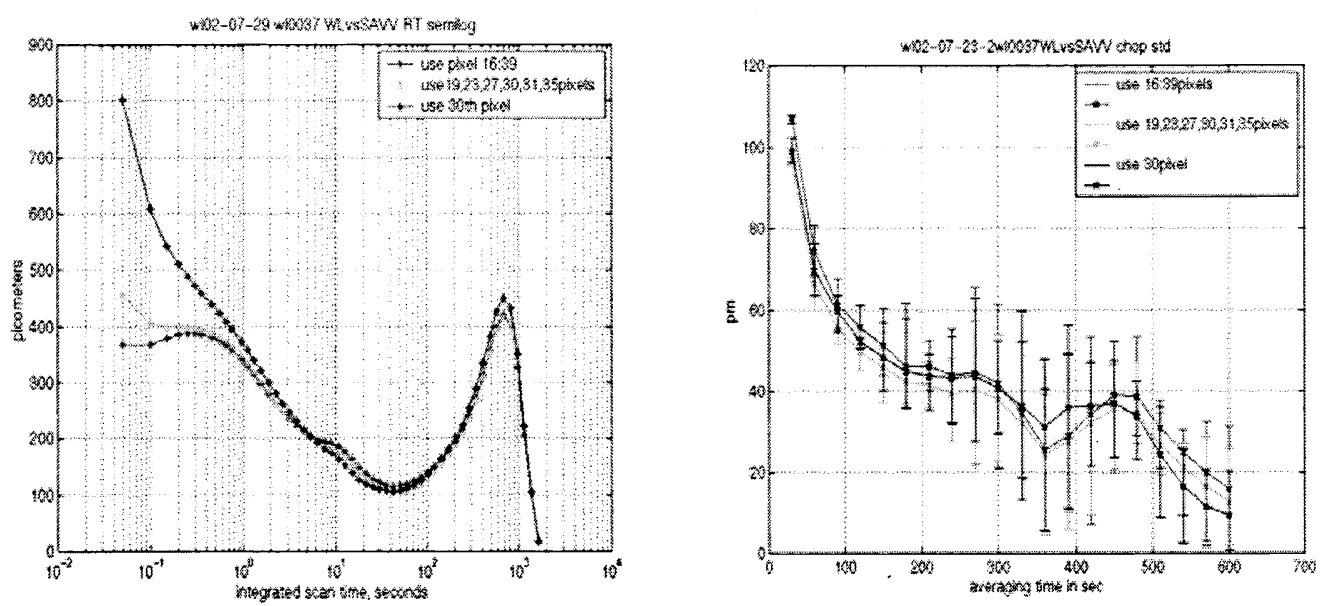

Fig. 10 Modified Allan deviation (left) and chop deviation (right) of OPD differences between white light and SAVV.

In Fig. 11, we explore the possible degradation of performance at increasing slew (or wait) times. We can still achieve the accuracy of $100 \mathrm{pm}$ at 10 chops with $30 \mathrm{sec}$ integration time and $60 \mathrm{sec}$ slew (or wait) time. This is an important feature for our future field dependent tests.

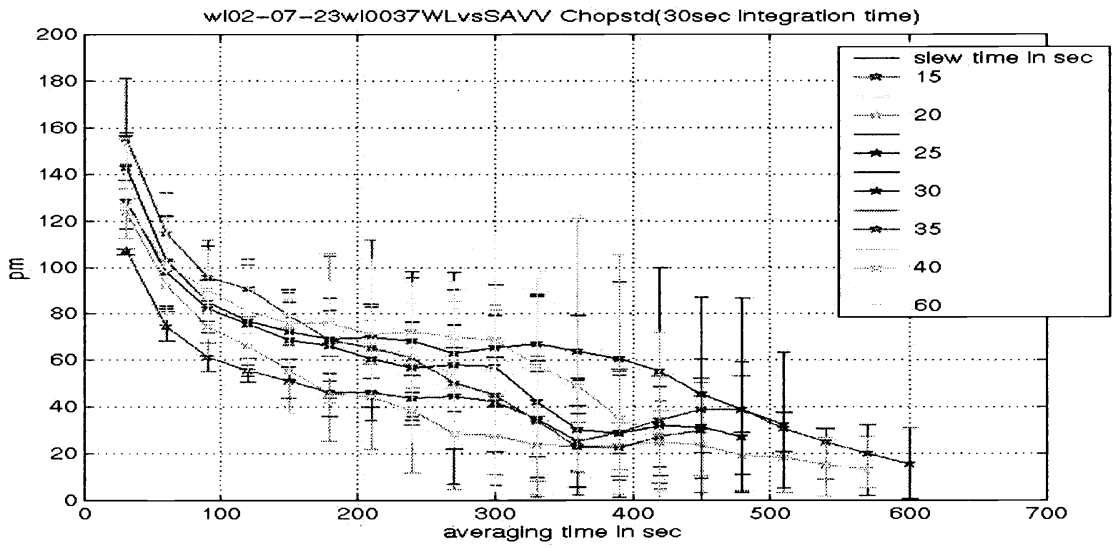

Fig. 11. The performance dependence on slew time.

\section{Conclusions}

We have used the MAM testbed to establish and validate performance and diagnostic metrics for the SIM flight system instrument. We have also demonstrated that our baseline fringe estimation algorithm provides adequate accuracy for the current MAM experiments. At lower light levels and less accurate calibration, we may have to use other algorithms instead. 


\section{REFERENCES}

1. Andreas C. Kuhnert, Stuart B. Shaklan, Tsae-Pyng Shen, "First Tests of the Interferometer in the Micro-arcsecond Metrology (MAM) Testbed", SPIE (Munich 2000).

2. Stuart B. Shaklan, Tsae-Pyng Shen, Mark H. Milman, and Andreas C. Kuhnert, "Picometer Measurement of White-Light Interference Fringes", ODIMAP III conference (Italy 2001).

3. F. Zhao, R. Diaz, G. M. Kuan, N. Sigrist, Y. Beregovski, L. L. Ames, K. Dutta, "Internal metrology beam launcher development for the space interferometry mission," SPIE conference Interferometry in Space, vol. 4852, 2002.

4. B. E. Hines, C. E. Bell, R. Goullioud, R. Spero, G. W. Neat, T. J. Shen, E. E. Bloemhof, M. Shao, J. Catanzarite, M. Regehr, R. Machuzak," Micro-arcsecond metrology (MAM) testbed overview", SPIE conference Interferometry in Space, vol. 4852, 2002.

5. K. Creath, "Phase-Measurement Interferometry techniques", Progress in Optics XXVI, Elsever Science Publishers, 1988.

6. D.W. Allan, "Statistics of Atomic Frequency Standards", Proc. of IEEE, V.54, p.221, 1966. J.von Neuman, R.H. Kent, H.R. Bellinson, and B.I. Hart, "The Mean Square Successive Difference”, Ann. Math. Stat. V.12, p. 153, 1942.

7. G. Neat, "MAM testbed and its relationship to SIM," SPIE conference Interferometry in Space, vol. 4852, 2002.

8. Mark H. Milman, Joe Catanzarite, and Slava G. Turyshev, "Effect if wave-number error on the computation of path-length delay in white-light interferometry", Applied Optics, V. 41, No. 23, p.1, 2002.

9. Mark H. Milman and Scott Basinger, "Error sources for white-light fringe estimation at low light levels", Appl. Opt. V. 41, p.2655, 2002.

10. R. Goullioud and E. Bloemhof, " Alignment of the MAM testbed to enable micro-arcsecond astrometry performance for SIM," SPIE conference Interferometry in Space, vol. 4852, 2002.

\section{ACKNOWLEDGMENT}

The research described was performed at the Jet Propulsion Laboratory, California Institute of Technology, under a contract with the National Aeronautics and Space Administration. Special thanks to Rich Machuzak for his skilled, tireless, and ever-cheerful operation of the MAM experiments. Thanks to Renaud Goullioud, Feng Zhao, Jeff Yu and Mike Shao for conversations providing helpful insight. Thanks to the entire team of people at JPL who have helped with MAM, and to our partners at Lockheed Martin for their contributions to MAM. 